\title{
ARTICLE Cognitive rigidity and BDNF-mediated frontostriatal glutamate neuroadaptations during spontaneous nicotine withdrawal
}

\author{
Robert D. Cole ${ }^{1}$, Matty Zimmerman ${ }^{1}$, Anastasia Matchanova ${ }^{1}$, Munir Gunes Kutlu ${ }^{2}$, Thomas J. Gould ${ }^{2}$ and Vinay Parikh ${ }^{1}$
}

\begin{abstract}
Cognitive flexibility is the ability to switch strategic responses adaptively in changing environments. Cognitive rigidity imposed by neural circuit adaptations during nicotine abstinence may foster maladaptive nicotine taking in addicts. We systematically examined the effects of spontaneous withdrawal in mice exposed to either nicotine $(6.3 \mathrm{or} 18 \mathrm{mg} / \mathrm{kg} / \mathrm{day})$ or saline for 14 days on cognitive flexibility using an operant strategy set-shifting task. Because frontostriatal circuits are critical for cognitive flexibility and brain-derived neurotrophic factor (BDNF) modulates glutamate plasticity in these circuits, we also explored the effects of nicotine withdrawal on these neurochemical substrates. Mice undergoing nicotine withdrawal required more trials to attain strategyswitching criterion. Error analysis show that animals withdrawn from both nicotine doses committed higher perseverative errors, which correlated with measures of anxiety. However, animals treated with the higher nicotine dose also displayed more strategy maintenance errors that remained independent of negative affect. BDNF mRNA expression increased in the medial prefrontal cortex (mPFC) following nicotine withdrawal. Surprisingly, BDNF protein declined in mPFC but was elevated in dorsal striatum (DS). DS BDNF protein positively correlated with perseverative and maintenance errors, suggesting mPFC-DS overflow of BDNF during withdrawal. BDNF-evoked glutamate release and synapsin phosphorylation was attenuated within DS synapses, but enhanced in the nucleus accumbens, suggesting a dichotomous role of BDNF signaling in striatal regions. Taken together, these data suggest that spontaneous nicotine withdrawal impairs distinct components of cognitive set-shifting and these deficits may be linked to BDNF-mediated alterations in glutamate signaling dynamics in discrete frontostriatal circuits.
\end{abstract}

Neuropsychopharmacology (2020) 45:866-876; https://doi.org/10.1038/s41386-019-0574-6

\section{INTRODUCTION}

Despite the known health concerns related to tobacco smoking and the availability of pharmacotherapies to aid in smoking cessation, relapse and failed cessation rates remain frustratingly high. Substantial evidence indicates that abrupt cessation from smoking produces a withdrawal syndrome that comprises somatic, affective, and cognitive components, due to disruption of neuroadaptations produced when nicotine, a primary addictive ingredient of tobacco, is removed from the system [1-4]. Contemporary models of addiction have emphasized that withdrawal-related alterations in cognition may predict relapse, and that distinct cognitive processes may be targeted for the treatment of nicotine dependence [5-7].

A plethora of studies conducted in abstinent smokers and animals undergoing withdrawal from chronic nicotine reported impairments in sustained attention, working memory, response inhibition, and hippocampus-dependent contextual learning processes [8-12]. Additionally, cognitive function predicted successful cessation in abstinent smokers [13]. Although these empirical studies supported the idea that distinct cognitive substrates could be considered endophenotypes of nicotine dependence, they remain limited in providing a clear neurobiological framework for targeting a specific cellular-cognitive mechanism to decrease relapse rates in smokers.
Cognitive flexibility, defined as the ability to switch strategic responses adaptively between changing environmental stimuli, is a fundamental component of fronto-executive function. Executive dysfunction is hypothesized to contribute to impairment in behavioral control and inability to consistently abstain from drugs in addicts [14]. Although previous research has shown that acute nicotine exposure facilitates perceptual set-shifting in rodents [15] while strategy switching remained intact with chronic nicotine exposure [16, 17], how nicotine withdrawal affects cognitive flexibility and neural circuits that subserve it remains elusive.

Preclinical and clinical evidence indicates that the integrity of frontostriatal circuits is crucial in flexible decision-making [18, 19]. In rodents, specific inactivation of either medial prefrontal cortex (mPFC) or discrete striatal regions such as dorsal striatum (DS) and nucleus accumbens (NAc) impaired performance in behavioral set-shifting tasks [20-23]. Although there is some evidence that transient inactivation or lesioning of ventral hippocampus in neonatal rats also impair cognitive flexibility during adulthood, such deficits have been ascribed to the developmental dysregulation of the PFC $[24,25]$.

mPFC projection neurons provide glutamatergic inputs to the DS and NAc, and optimal glutamate signaling is essential to support cognitive flexibility [26-28]. Extensive evidence suggests

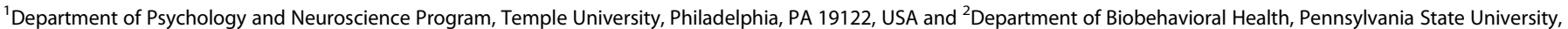
University Park, PA 16802, USA

Correspondence: Vinay Parikh (vinay.parikh@temple.edu)

Received: 14 July 2019 Revised: 13 October 2019 Accepted: 14 November 2019

Published online: 21 November 2019 
that brain-derived neurotrophic factor (BDNF), via its interactions with high-affinity tropomyosin-related kinase B (trkB) receptors, regulates plasticity at the striatal glutamatergic synapses [29-31]. Moreover, we previously reported that BDNF modulates glutamate release in the DS in an inverted-U fashion [32], and that cognitive performance and BDNF protein in the MPFC-DS circuit was altered in mecamylamine-precipitated withdrawal [33]. This suggests that nicotine withdrawal-associated circuit adaptations and functional deficits may involve alterations in BDNF signaling.

Here we hypothesize that cognitive rigidity imposed by withdrawal due to abrupt cessation of nicotine is associated with BDNFmediated alterations in glutamatergic signaling in discrete frontostriatal regions. To this end, we systematically assessed the effect of spontaneous withdrawal from chronic nicotine administered at two different doses (6.3 and $18 \mathrm{mg} / \mathrm{kg} /$ day) in mice performing an operant-based strategy set shift task. BDNF expression within frontostriatal circuits was examined to determine the association between withdrawal-related alterations in cognitive performance and negative affective components of withdrawal that are considered to be critical determinants of nicotine dependence [2]. Lastly, striatal glutamate responsivity to BDNF was assessed using in vivo amperometric recordings to determine the effect of nicotine withdrawal on neurochemical circuit adaptations.

\section{MATERIALS AND METHODS}

Subjects

Sixty-nine young-adult (8-10 weeks old) male C57BL/6J mice procured from Jackson Laboratory (Bar Harbor, ME) were housed in a humidity/temperature-controlled colony room with a $12 \mathrm{~h}$ light/dark cycle (lights on at 07:00). For behavioral studies, mice were individually housed and water-restricted to $5 \mathrm{~min}$ of water per day. All behavioral training and testing took place 7 days/week between 9:00 and 16:00 h. Food (PMI LabDiet) was available ad libitum throughout the experiments. For all other studies, mice remained grouped housed. All experimental procedures were approved by the Institutional Care and Use Committee (IACUC) of Temple University and were in accordance with the National Institute of Health guidelines.

Operant behavioral training and testing

Operant studies were conducted in Modular chambers (MED Associates; St. Albans, VT) equipped with a SmrtCtrl interface running MED-PC IV software on Dell PC (Optiplex 960). Mice were trained in a strategy set-shifting task based on previous work from our laboratory $[16,17,32,33]$. This task was adapted from a behavioral flexibility task in rats and emulates features of the Wisconsin Card Sorting Task that asseses human cognitive flexibility [34,35]. Briefly, animals were autoshaped and pretrained on a fixed-ratio 1 schedule of reinforcement and then progressed to the spatial discrimination phase that required them to adopt an egocentric response strategy to achieve rewards $(0.066 \%$ of saccharin solution). A lever press on the assigned lever (e.g. right lever for right assignment) was scored as a "correct response" and was rewarded. Responses on the incorrect lever (i.e. left lever in this case) were not rewarded and resulted in a "timeout" (negative punishment) phase characterized by a $5 \mathrm{~s}$ extinguishing of the house light. After attaining criterion (80\% correct responses for three consecutive days), the animals were assessed for set-shifting that required a switch from a spatial discrimination-based strategy (lever assignment) to a visual discrimination-based strategy to attain rewards (Fig. 1a). During this phase, contingencies were altered to require mice to discern the lever with an activated cue light, which remained pseudorandom. Mice remained on the strategy set-shifting phase until they either reached criterion performance ( $\geq 80 \%$ correct responding for three consecutive days) or 2 weeks whichever was earlier. Each behavioral session consisted of 30 trials with an ITI of $9 \pm 3 \mathrm{~s}$. The number of correct responses, errors, omissions, response latencies, and reward retrieval latencies were obtained for each behavioral session. Response accuracies were calculated for each session using the formula: correct responses/(correct + incorrect responses) $\times 100$. The behavioral components of set-shifting were characterized by categorizing error responses as either perseverative or maintenance. A perseverative error occurred if the animal responded to the lever assigned during lever discrimination (e.g. right lever; Fig. 1a) without the presence of the visual cue illuminated above it on $\geq 60 \%$ of trials within a session. Depending on the training performance in the preceding session, this error was scored as a regressive error if the animal makes $<60 \%$ incorrect responses in subsequent sessions. At this point, the animals were considered to be inhibiting the previously learned strategy and acquiring the new strategy. Never-reinforced errors occur if an animal responds on the opposite lever assigned during lever discrimination while the visual cue is presented from the opposite side. Both regressive and never-reinforced errors were categorized as "maintenance errors" as they reflect an index of the acquisition/execution of a new strategy. For further task details, refer to Supplementary Information.

Osmotic minipump surgeries, nicotine withdrawal, and experimental design

Animals that attained pretraining criterion were randomly assigned to saline, low-dose nicotine, and high-dose nicotine groups ( $N=8 /$ group). Mini-osmotic pumps (model 1002, DURECT Corp., Cupertino, CA) designed to administer nicotine/saline solution at a rate of $0.25 \mu \mathrm{L} / \mathrm{h}$ for 14 days were implanted subcutaneously below the back region in isoflurane-anesthetized mice. Nicotine (Sigma-Aldrich, St. Louis, MO) was administered either at $6.3 \mathrm{mg} / \mathrm{kg} /$ day (low dose) or at $18 \mathrm{mg} / \mathrm{kg} /$ day (high dose); doses are reported as free base. Similar doses in mice produced plasma nicotine or cotinine (a metabolite of nicotine) levels in the range reported in human smokers $[8,16,36,37]$. Once the animals regained pretraining criterion post-surgery, they progressed through the spatial discrimination phase of the task. Following acquisition, the animals remained on this phase till the 14-day chronic nicotine/vehicle exposure period (Fig. 1b).

Spontaneous nicotine withdrawal was induced by surgical removal of osmotic minipumps. Behavioral testing began on the 15th day during which the animals were switched to the visual discrimination (i.e. set-shifting) phase. All animals were monitored for somatic signs of withdrawal that included headshakes, paw tremors, retropulsion, writhing, scratching, backing, piloerection, and Straub tail on the first (WD1), third (WD3), and seventh (WD7) day following pump removal. Mice were also examined for negative affective symptoms of nicotine withdrawal by monitoring the anxiety-like behavior using the elevated plus maze as described previously $[38,39]$. Each testing session began with the mouse being placed in the center of the maze. An entry into an arm was scored when all four paws and head entered into the arm. Mice are allowed to explore the maze for $5 \mathrm{~min}$. The percentage of time spent on the open arms was calculated as the percentage of the total time the mouse spent on the maze. EPM testing also occurred on WD1, WD3, and WD7. Brain tissues were removed for neurochemistry after the last behavioral session to determine the consequences of nicotine withdrawal on BDNF signaling in the MPFC-DS and mPFC-NAc circuits and how it relates to behavioral changes (Fig. 1b, c). For behavioral control experiments, a separate cohort of animals $(N=12)$ was used to assess the effect of spontaneous nicotine withdrawal on initial learning that occurred prior to set-shifting testing, i.e. spatial discrimination. Moreover, initial discrimination was also assessed in saline- and nicotine-exposed mice prior to the induction of withdrawal and subsequent testing on the strategy set-shifting phase (see Supplementary Information for more details on the control experiments). 
A spatial discrimination (right assignment)
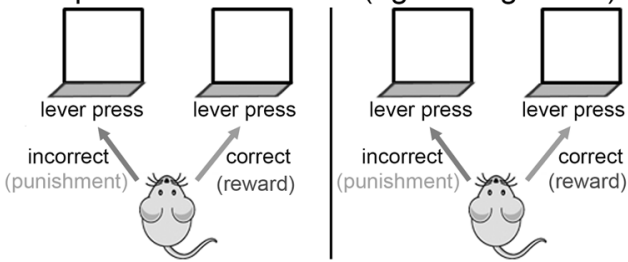

$\mathrm{B}$

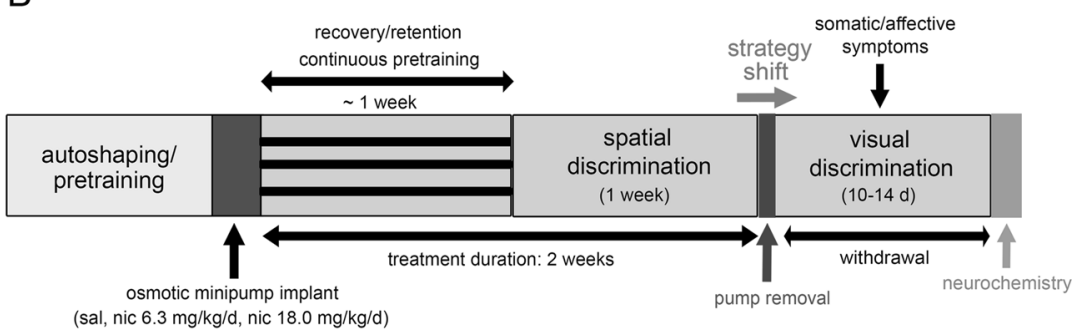

visual discrimination

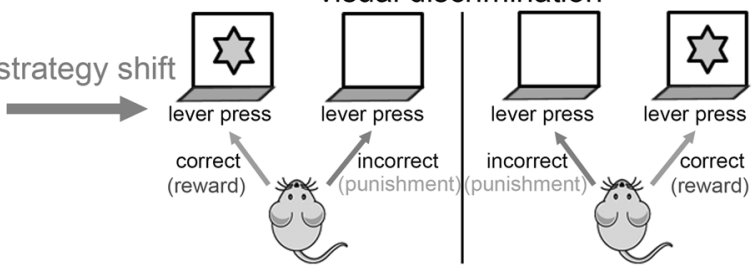

C

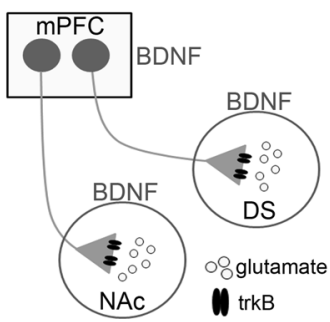

Fig. 1 Schematics of the strategy set-shifting task and experimental design. a Mice were initially autoshaped and pretrained to acquire a lever press response within a specified period of time to receive a reward (sweetened water). Animals that reached pretraining criterion were progressed to the spatial discrimination phase in which they were required to adopt an egocentric response strategy to achieve rewards. During each trial, animals were presented with both right and left levers A lever press on the assigned lever (right assignment in this case; lever assignment was counterbalanced within a group) was scored as a "correct response" and was followed by reward delivery. Response on the incorrect lever (left lever in this case) was not rewarded and resulted in a punishment (timeout) phase characterized by a $5 \mathrm{~s}$ extinguishing of the house light. After reaching criterion performance, mice progressed to the set-shifting (visual discrimination) phase. In this phase, mice were required to abandon the previously reinforced strategy in favor of a current visual cue-based strategy. A lever press response on the lever associated with the illuminated light was scored as a "correct response' and was subsequently rewarded. Lever responses to the lever opposite of the light cue were scored as an "error" and resulted in punishment ( $5 \mathrm{~s}$ timeout). b After attaining pretraining criterion, mice were implanted with mini-osmotic pumps delivering saline, 6.3 or $18 \mathrm{mg} / \mathrm{kg} /$ day nicotine for 14 days. After 1 week of post-surgery recovery and behavioral retention, mice progressed to the spatial discrimination phase. Animals remained on this phase for 7 days during which they attain criterion. Following the last training session on the 14th day of solution delivery, pumps were removed and spontaneous withdrawal was induced. Mice were then tested on strategy set-shifting (visual discrimination) $\sim 24 \mathrm{~h}$ after pump removal and remained on this phase until either criterion performance was reached or 2 weeks whichever was earlier. Tissues were removed for neurochemistry studies after the last behavioral session. c Schematic illustrating discrete components of the frontostriatal circuits examined for the consequences of nicotine withdrawal on BDNF signaling and glutamatergic transmission.

Neurochemistry

$q P C R$. The expression levels for BDNF exon-IV transcript (driven by neuronal activity), and BDNF exon-IX transcript, a measure of total BDNF transcription [40], were measured using a quantitative reverse-transcription polymerase chain reaction (qPCR) as described previously [41]. Total RNA was isolated from mPFC using the AllPrep DNA/RNA Mini Kit (Qiagen, Hilden, Germany). qPCR was performed using the Power SYBR Green RNA-to-CT 1step kit (Applied Biosystems, Austin, TX) and the expression levels were normalized using GAPDH as a housekeeping gene using the $\triangle \mathrm{Ct}$ method (see Supplementary Information for primers and other details).

Immunoblotting. Tissues from the mPFC, DS, and NAc were homogenized in an ice-cold $5 \mathrm{mM}$ HEPES lysis buffer containing a protease inhibitor cocktail. Proteins were separated using SDSPAGE electrophoresis and blots were transferred onto PVDF membranes to detect BDNF/proBDNF and trkB protein bands. To determine the effects of BDNF on synapsin phosphorylation in presynaptic striatal terminals, immunoblotting was conducted on synaptosomes isolated from the DS and NAc of a separate cohort of animals that underwent saline/nicotine ( $N=4 /$ group) withdrawal (refer Supplementary Information for method details and densitometric analysis).

In vivo amperometric recordings of glutamatergic transmission The effects of nicotine withdrawal on glutamate dynamics in the target striatal regions of mPFC projections (i.e. NAc and DS) were examined using in vivo amperometric recordings (Fig. 1c). For these experiments, a separate cohort of adult mice $(N=5-6$ per group) subjected to either saline or nicotine $(18 \mathrm{mg} / \mathrm{kg} /$ day) withdrawal were used. Based on the time series analysis of response accuracies in our behavioral experiments (see Results), the deficits appeared more prominent during the first week of withdrawal. Therefore, all recordings were planned within 3-7 days following withdrawal. Ceramic-based microelectrode arrays (Quanteon LLC, Nicholasville, KY) were coated with glutamate-oxidase as described previously [32, 42, 43] and the calibrated electrodes were implanted into the DS and NAC of urethane-anesthetized animals. Depolarization- and BDNFinduced glutamate release was recorded by applying local pulses of potassium $(\mathrm{KCl} 70 \mathrm{mM} ; 100 \mathrm{~nL})$ and BDNF (10 ng; $100 \mathrm{~nL})$, respectively. In addition, alterations in extracellular glutamate levels were also measured following the local application of nicotine $(1 \mathrm{mM} ; 100 \mathrm{~nL})$. All recordings were conducted at $2 \mathrm{~Hz}$ by applying $+0.7 \mathrm{~V}$ fixed potential and data were digitized using a FAST-16 potentiostat (Quanteon). Electrochemical currents from three signals were averaged and analyzed with respect to the peak signal amplitudes (refer Supplementary Information for procedural details).

Statistical analysis

All behavioral, biochemical, and amperometry data were analyzed using one-way ANOVA to compare group differences between saline, lower and higher nicotine withdrawal groups unless otherwise noted. A mixed-factor ANOVA was used to analyze response accuracy, errors, correct. and incorrect latencies utilizing the first 10 days after behavioral shifting as a within-groups factor 
and nicotine treatment as between-groups factor. Post hoc tests were applied using Tukey's HSD. Pearson's correlation coefficient was calculated for all correlative analyses. For all statistical tests, $p$ values $\leq 0.05$ were considered statistically significant.

\section{RESULTS}

Spontaneous nicotine withdrawal impairs strategy set-shifting Induction of spontaneous nicotine withdrawal in mice was confirmed by the examination of somatic symptoms (Supplementary Fig 1). Assessment of cognitive performance revealed that mice undergoing nicotine withdrawal required more trials to attain strategy set-shifting criterion $(F(2,22)=16.11, p<0.001$, $\eta^{2}=0.58$; Tukey's HSD: $p<0.001$ for both nicotine doses; Fig. 2a) and committed higher number of errors $(F(2,22)=6.631, p<0.01$, $\eta^{2}=0.37$; Tukey's HSD: $p<0.02$ for both nicotine doses; Fig. $2 b$ ). The set-shifting errors committed for 10 behavioral sessions are depicted in Fig. 2c. In general, the number of errors declined with increasing testing sessions (main effect of sessions: $F(9,189)=$ $16.28, p<0.001, \eta^{2}=0.44$ ). Moreover, significant differences in set-shifting errors were observed between the treatment groups
$\left(F(2,21)=16.28, p<0.001, \eta^{2}=0.61\right)$. However, the effect of training sessions did not interact with treatment conditions $(F(18$, $\left.189)=1.73, p=0.11, \eta^{2}=0.14\right)$. Further analyses by error types uncovered that perseverative errors remained significantly higher in nicotine withdrawal mice $\left(F(2,22)=5.26, p=0.01, \eta^{2}=0.32\right.$; Tukey's HSD: $p<0.04$ for both doses vs saline withdrawal; Fig. $2 d$ ). Maintenance errors differ significantly between treatment groups $\left(F(2,22)=5.54, p=0.01, \eta^{2}=0.42\right.$; Fig. 2e). Upon subsequent analyses, no differences were uncovered between the saline and low-dose nicotine groups $(p=0.99)$, but the higher-dose nicotine group committed significantly more maintenance errors $(p=0.02$ vs both saline and low-dose nicotine, respectively). The response accuracies analyzed for 10 behavioral sessions show sessiondependent learning (main effect: $F(9,189)=29.78, p<0.001$, $\left.\eta^{2}=0.59\right)$, and this effect interacted with treatment groups ( $F$ (18, $189)=1.81, p=0.03, \eta^{2}=0.15$; Fig. 2f). Post hoc analysis show that saline-withdrawal mice were faster in attaining strategy set shift criterion compared to nicotine-exposed animals (both doses; $p<0.01$ for WD1-9). Although accuracies remained similar between the two nicotine doses $(p>0.05)$, differences in error types (noted above) might reflect that learning strategy was
A

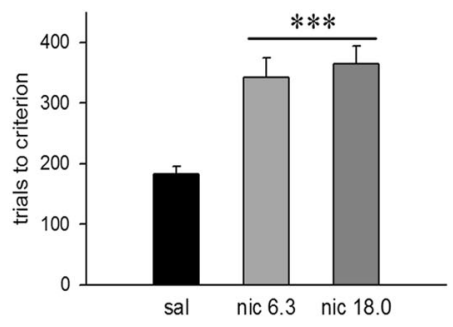

D

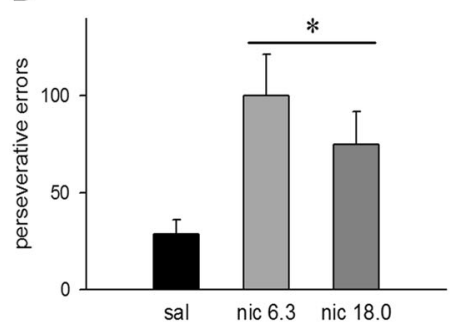

G

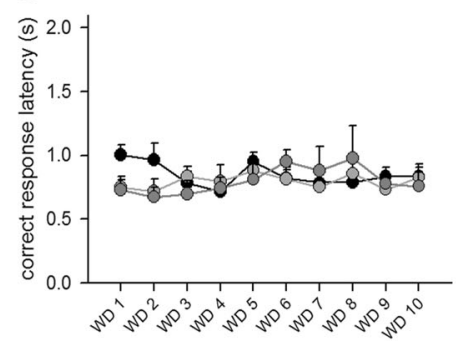

B

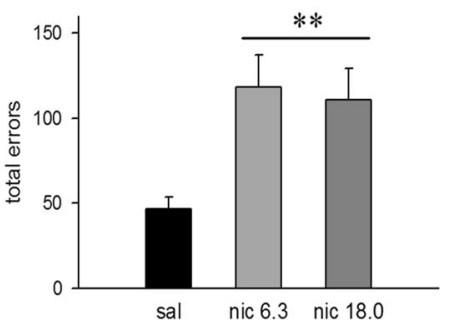

E

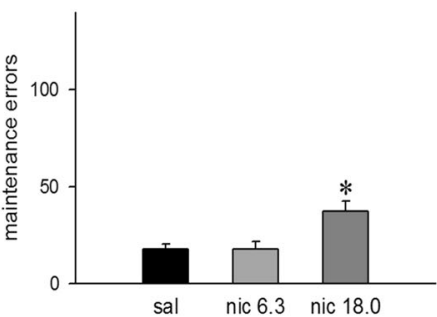

$\mathrm{H}$

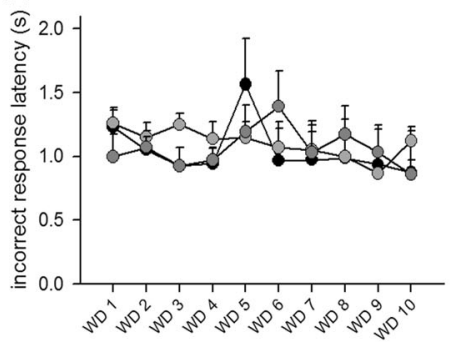

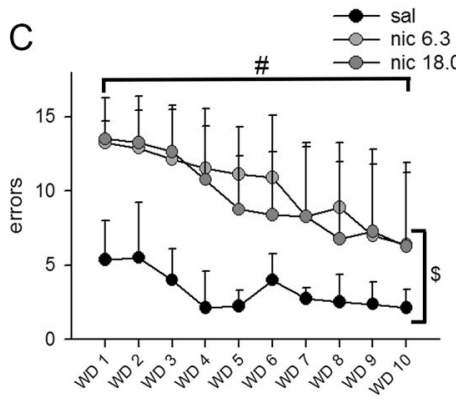

F

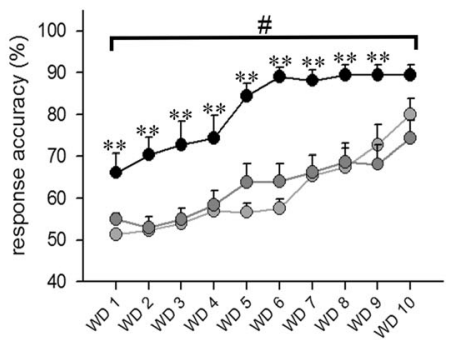

I

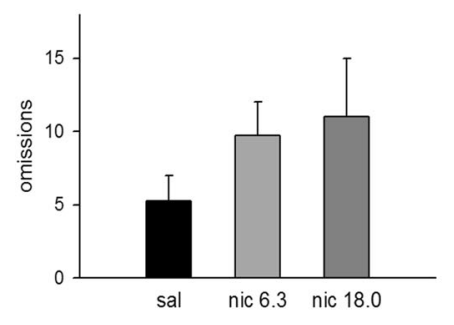

Fig. 2 Spontaneous nicotine withdrawal strategy set-shifting performance. Mice experiencing spontaneous withdrawal following the removal of mini-osmotic pumps that delivered nicotine (both doses: 6.3 and $18 \mathrm{mg} / \mathrm{kg} / \mathrm{d}$ ) for 2 weeks required more trials to reach criterion performance (a) and committed more total errors (b) as compared to saline-exposed mice. Although set-shifting errors incrementally reduced as the animals received more testing sessions; they remain significantly different between the treatment groups (c). Further investigation of error types revealed that withdrawal from either dose of nicotine significantly increased perseverative errors (d); however, maintenance errors significantly increased only in mice that were withdrawn from the higher dose of nicotine (e). Response accuracies for 10 testing sessions depict delayed acquisition of the strategy set shift in nicotine withdrawal animals (f). Correct $(\mathbf{g})$ and incorrect (h) response latencies remained similar across all testing sessions between the groups. The omissions remained similar across all withdrawal conditions (i). Data are mean \pm SEM ( $n=8$ mice per group). Trials to criterion, total errors, perseverative errors, maintenance errors, and omissions were analyzed using oneway ANOVA. Analysis of errors and response latencies for 10 behavioral sessions was conducted using mixed-factor ANOVA. \# $p<0.001$ (main effect of session); ${ }^{\$} p<0.001$ (main effect of treatment); ${ }^{*} p<0.05 ;{ }^{* *} p<0.01 ;{ }^{* * *} p<0.001$ (post hoc tests). 
impacted more in the high-dose nicotine group. Comparisons of response latencies for both correct and incorrect trials show no significant differences between the treatment groups (all $F<1.59$, all $p>0.12$, all $\eta^{2}>0.04$; Fig. $\left.2 g, h\right)$. Moreover, omissions remained unaffected by nicotine withdrawal $\left(F(2,22)=1.05, p=0.36, \eta^{2}=\right.$ 0.09 ; Fig. 2i). Behavioral control experiments to assess the effects of spontaneous nicotine withdrawal on spatial discrimination (the initial learning phase of the set-shifting task) did not reveal any significant differences in trials and errors to criterion (see Supplementary Results and Supplementary Fig 2). Likewise, spatial discrimination performance during chronic nicotine exposure did not differ significantly as compared to saline-exposed mice prior to the induction of withdrawal and set-shifting testing (Supplementary Fig 2). Together, these data indicate that the behavioral effects were specific to the set-shifting performance and occurred only during spontaneous nicotine withdrawal.

Frontostriatal BDNF expression and nicotine withdrawal-related alterations in cognition

qPCR analysis revealed significant group differences for both BDNF exon-IV $\left(F(2,23)=10.37, p=0.001, \eta^{2}=0.51\right)$ and BDNF exon-IX (total BDNF; $F(2,23)=5.85, p=0.01, \eta^{2}=0.36$ ) mRNA, respectively. Post hoc comparisons indicated that withdrawal from the higher-dose nicotine increased BDNF exon-IV transcription significantly ( $p=0.03$ vs saline; Fig. 3a). However, exon-IV transcripts significantly decreased in lower-dose nicotine group ( $p=0.04$ vs saline). When comparing BDNF exon-IX (Fig. 3b), withdrawal from the higher-dose nicotine increased total BDNF transcription ( $p=0.02$ vs saline; $p=0.004$ vs low nicotine). Immunoblot analysis of BDNF protein (mature form) in the MPFC revealed significant differences between experimental groups $\left(F(2,21)=4.41, p=0.03, \eta^{2}=0.32\right)$. Post hoc tests uncovered a significant reduction in $\mathrm{MPFC}$ BDNF protein in higher-dose nicotine withdrawal mice $(p=0.01$; Fig. $3 c)$. On the contrary, a significant increase the BDNF protein was noted in the DS of these animals $\left(F(2,21)=5.244, p=0.01, \eta^{2}=0.32\right.$; Tukey's HSD: $p=$ 0.01 vs saline; Fig. $3 d$ ). Correlation analysis revealed a trend for a negative association for BDNF protein levels between PFC and DS (Pearson's $r=-0.39, p=0.06$ ). Surprisingly, no changes in BDNF protein were observed in the NAc following nicotine withdrawal $\left(F(2,21)=0.73, p=0.49, \eta^{2}=0.06\right.$; Fig. 3e). The expression of BDNF high-affinity receptor trkB remained unaltered in both striatal regions (DS: $F(2,21)=1.43, p=0.26, \eta^{2}=0.06$; NAc: $F(2,21)=0.01, p=0.99, \eta^{2}=0.01$; Fig. 3f). Pro-BDNF levels did not differ in frontostriatal regions across the treatment groups (Supplementary Fig 3).

To examine whether alterations in BDNF protein is associated with specific components of strategy set shifting, we conducted correlation analyses between region-specific BDNF changes and error types. A significant negative correlation was observed between prefrontal BDNF expression and preservative errors (Pearson's $r=-0.49, p=0.01$; Fig. 3g), but not for maintenance errors (Pearson's $r=-0.26, p=0.20$; Fig. 3j). Conversely, DS BDNF protein was positively associated with both perseverative (Pearson's $r=0.45, p=0.02$; Fig. 3h) and maintenance errors (Pearson's $r=0.43, p=0.03$; Fig. 3k), respectively. No significant correlations were found between NAc BDNF expression and the two error types (both $p>0.31$; Fig. $3 \mathrm{i}$, I). It should be noted that lower sample size constrained our ability to derive any meaningful information by separately analyzing the correlation data by groups. Therefore, we examined how treatments would have impacted the linearity of association between the cognitive measures and BDNF levels by conducting multiple linear regression analyses using treatments (saline, low-dose nicotine, high-dose nicotine) as the categorical variables (codes for treatment 1: $0,1,0$; codes for treatment 2: $0,0,1)$. Our results indicate that both PFC BDNF and DS BDNF levels in the low-dose nicotine withdrawal group (vs. saline) significantly accounted for the variation in perseverative errors (PFC BDNF: $t$ ratio $=-2.24 ; p$ $=0.03$; DS BDNF: $t$ ratio $=-2.44 ; p=0.02$ ). On the other hand, DS BDNF levels mice withdrawn from the high-dose nicotine (vs. saline) significantly accounted for the variation in maintenance errors ( $t$ ratio $=-2.46 ; p=0.02$ ).

Association of nicotine withdrawal-related negative affect with strategy set-shifting and frontostriatal BDNF

Anxiety-like behavior was examined after induction of withdrawal using mixed factors ANOVA. Significant main effect of the withdrawal day $\left(F(2,44)=8.22, p=0.001, \eta^{2}=0.29\right)$ and group $\left(F(2,22)=15.89, p<0.001, \eta^{2}=0.63\right)$ was noted on time spent in open arms in EPM. During WD1 and WD3, both nicotine doses significantly reduced the amount of time spent in the open arms compared to saline (WD1: $p<0.001$; WD3: $p<0.01$; Fig. 4a). On WD7, no significant differences in the EPM measure was observed between the groups (all $p>0.14$; Fig. 4a) illustrating that anxiety-like symptoms lasted $<1$ week following nicotine withdrawal. A significant negative correlation was uncovered between the time spent in open arms (EPM measure of anxiety) and perseverative errors (Pearson's $r=-0.41, p=0.04$; Fig. 4b). This association was not observed with the maintenance errors (Pearson's $r=-0.36, p=0.08$; Fig. 4c). Prefrontal BDNF positively correlated with the percent time spent in the open arms (Pearson's $r=0.40, p=0.05$; Fig. 4d). Surprisingly, an inverse relationship was observed between DS BDNF levels and the EPM measure (Pearson's $r=-0.50, p=0.01$; Fig. 4e). Multiple linear regression using treatments as categorical variables (as described above) revealed that withdrawal from both low- and high-dose nicotine significantly contributed to the association between EPM measures and perseverative errors (both doses; $p<0.002$ ), and PFC and DS BDNF levels, respectively (all $p<0.003$ ). No significant association was observed between NAc BDNF and anxiety-like behavior (Pearson's $r=-0.28, p=0.19$; Fig. 4f).

Altered responsivity of striatal glutamatergic signaling to BDNF during spontaneous nicotine withdrawal

In vivo amperometric recordings revealed that the amplitudes of potassium depolarization-evoked glutamate signals did not significantly differ between saline and nicotine withdrawal mice in either the DS $\left(F(1,10)=2.56, p=0.144, \eta^{2}=0.12\right.$; Fig. 5a) or NAC $\left(F(1,10)=0.76, p=0.405, \eta^{2}=0.1\right.$; Fig. 5b). However, BDNFelicited glutamate signals were significantly blunted in the DS of nicotine withdrawal mice $\left(F(1,10)=27.44, p=0.001, \eta^{2}=0.82\right.$ vs saline; Fig. 5c). Interestingly, BDNF-evoked glutamate release in the NAc significantly increased in mice spontaneously withdrawn from nicotine $\left(F(1,10)=9.62, p=0.015, \eta^{2}=0.5\right.$; Fig $5 d$ ). Separate experiments conducted in synaptosomes isolated from animals undergoing saline and nicotine withdrawal revealed parallel changes in BDNF-induced phosphorylation of synapsin in the two striatal regions (Supplementary Fig 4). Local application of nicotine significantly augmented glutamate release in the DS of nicotine withdrawal mice $(13.64 \pm 1.23 \mu \mathrm{M}$ vs $5.38 \pm 1.19 \mu \mathrm{M}$ in saline; $\left.F(1,10)=22.58, p=0.001, \eta^{2}=0.88\right)$ reflecting compensatory upregulation of $n A C h R s$ (presumably a7 nAChRs) that are localized on glutamatergic terminals [44]. Nicotine-evoked glutamate signals did not differ between nicotine withdrawal and saline mice in the $\operatorname{NAc}(F(1,10)=$ $0.78, p=0.40, \eta^{2}=0.15$ ).

\section{DISCUSSION}

Our data demonstrate that strategy set-shifting deficits associated with spontaneous nicotine withdrawal were specifically manifested as an inability to appropriately switch response choices from one learning rule to another, and not as a generalized deficit in the instrumental learning of response outcome associations. Nicotine withdrawal produced a robust increase in perseverative 
A

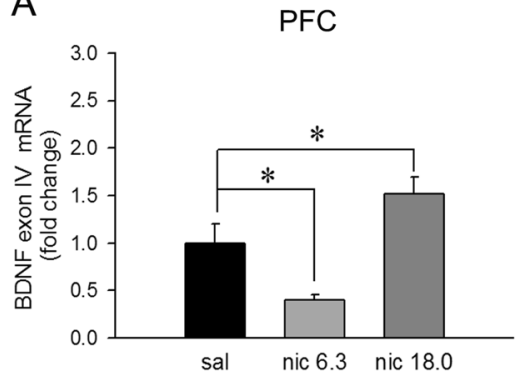

D

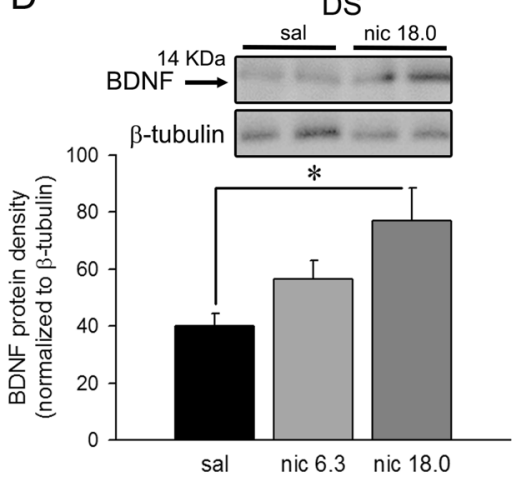

$\mathrm{G} \quad \begin{array}{ll}: & \text { sal } \\ : & \text { nic } 6.3 \\ 0 & \text { nic } 18.0\end{array}$
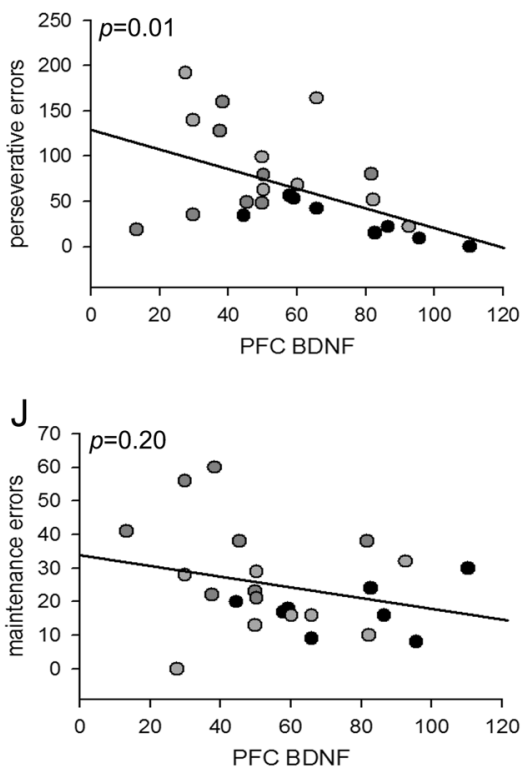

B

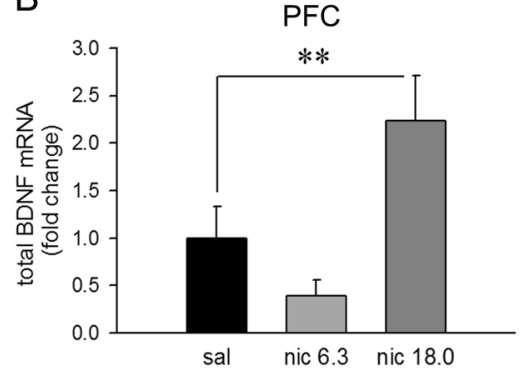

E

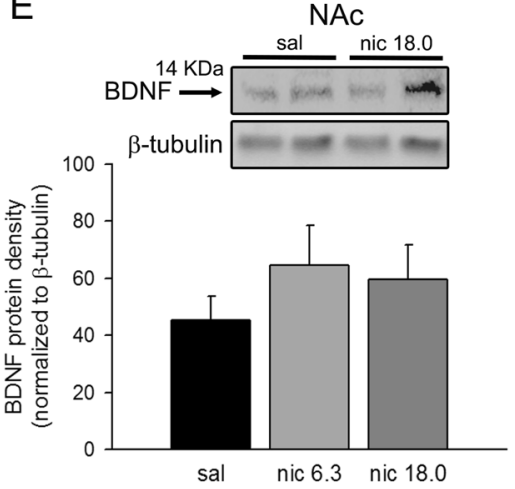

$\mathrm{H}$

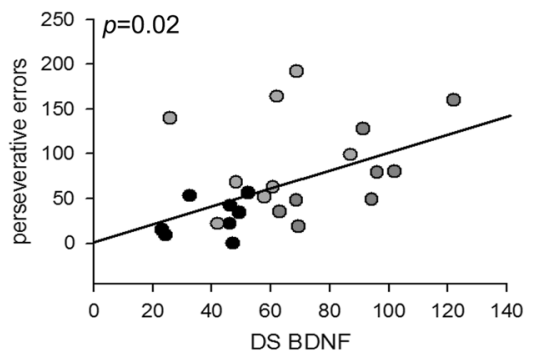

$\mathrm{K}$

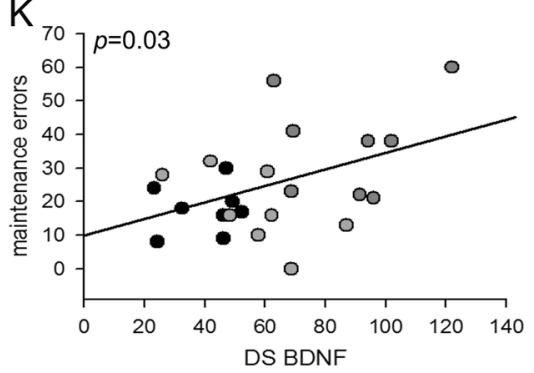

PFC
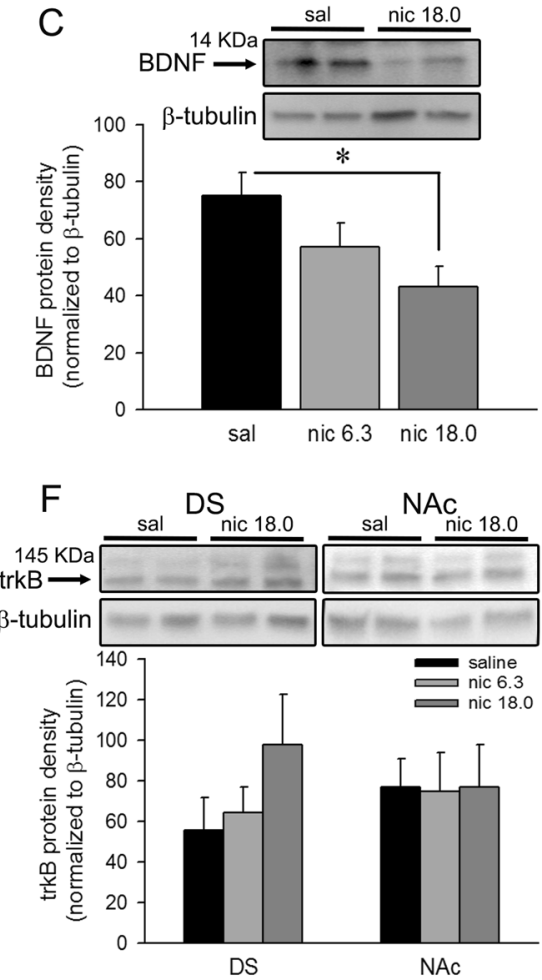

I
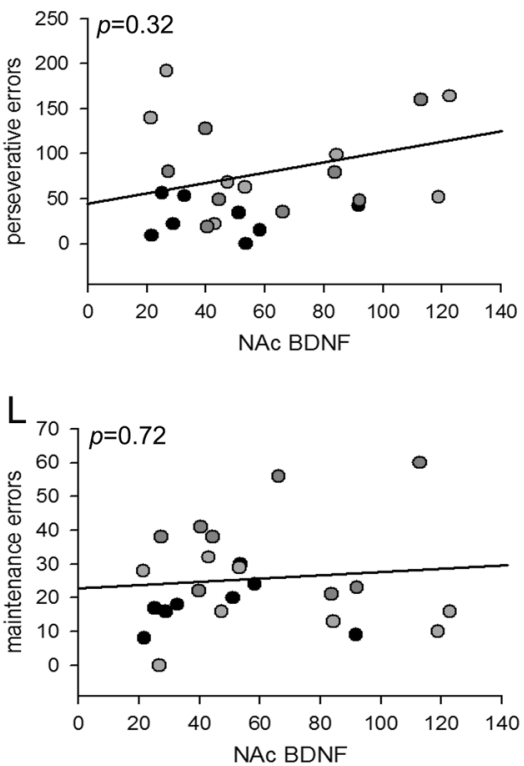

errors (errors that represent initial difficulty in switching to new response sets), and this effect remained similar between the two nicotine doses. Substance use interrupts the ability of individuals to inhibit prepotent responding to not only previously reinforcing stimuli [45], but also to responses not directly related to drug use [46]. Moreover, abstinence from nicotine has been shown to decrease disinhibition in both rodent as well as human studies $[47,48]$. The robust increase in perseverative errors may therefore indicate strategy shifting deficits due to generalized disruption of inhibitory control that may occur during the early stages of nicotine abstinence.
Interestingly, withdrawal induced by the higher-dose nicotine also increased strategy maintenance errors. In set-shifting tasks, these errors are categorized as non-perseverative errors (regressive and never-reinforced errors) where the subject randomly chooses either the previously acquired response rule or a rule that has never been reinforced, after the initial shift to the correct rule has occurred [23, 34]. Thus, disruption in maintaining new response sets (reflected as set-shifting deficits) might have occurred as a result of broader cognitive dysfunction associated with high-dose nicotine withdrawal. Indeed, non-perseverative errors observed in WCST relates to top-down attention, working 
Fig. 3 Nicotine withdrawal-related alterations in BDNF expression in the frontostriatal regions of task-performing mice. Bar charts illustrate the results of $\mathrm{qPCR}$ analysis of the neuronal activity-dependent BDNF exon-IV transcript (a) and BDNF exon IX for total BDNF mRNA (b) in the mPFC. All mRNA data is expressed as fold changes normalized to GAPDH. Mice withdrawn from the higher (18 mg/kg/day) dose of nicotine exhibited significantly higher expression of both BDNF exon IV and total BDNF mRNA as compared to saline. Surprisingly, the expression levels of exon-IV transcript significantly reduced following spontaneous withdrawal from the lower nicotine dose (6.3 mg/kg/day). This effect was not observed in the total BDNF mRNA. Immunoblot analyses showed reduced expression of BDNF protein (mature form of $\mathrm{BDNF}$ detected as a $14 \mathrm{kDa}$ immunoreactive band) in the $\mathrm{mPFC}$ of mice undergoing withdrawal from the higher nicotine dose as compared to saline-exposed animals (c). Conversely, BDNF protein levels were significantly elevated in the tissue homogenates prepared from the DS region of these mice (d). BDNF protein levels in the MPFC and DS regions remained unaltered at the lower nicotine dose. BDNF protein levels in the NAc remained indifferent between the treatment groups (e). The expression of the high-affinity BDNF receptor, TrkB, remained unaltered with nicotine withdrawal in the DS and NAc (f). All mRNA and protein data are expressed as mean \pm SEM ( $n=8$ mice/group) and analyzed using one-way ANOVA. ${ }^{*} p<0.05 ;{ }^{* *} p<0.01$ (post hoc tests). The results of Pearson's $r$ correlation analyses between BDNF protein levels and strategy set-shifting errors are depicted in $\mathbf{g - I}$. Prefrontal BDNF protein levels were negatively associated with perseverative errors $(\mathbf{g})$, while a completely opposite pattern was observed with DS BDNF levels (h). No association was observed between NAc BDNF protein levels and behavioral perseveration (i). While prefrontal BDNF levels did not correlate with the strategy maintenance errors (j), DS BDNF levels did show a positive correlation with this behavioral measure (k). NAc BDNF expression was not associated with strategy maintenance errors (I).

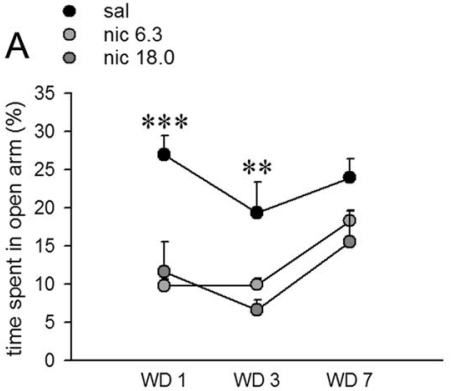

D

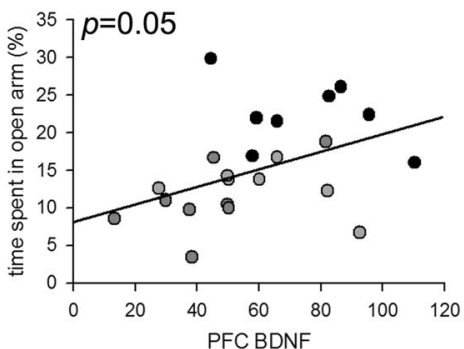

B

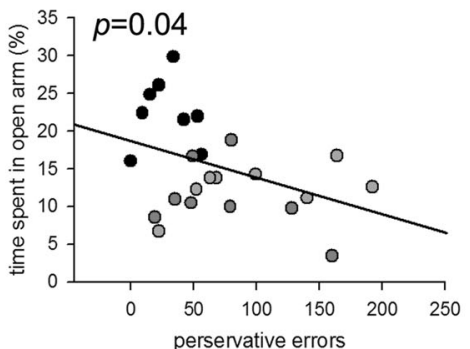

$\mathrm{E}$

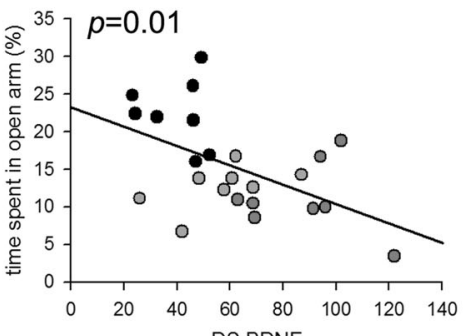

C

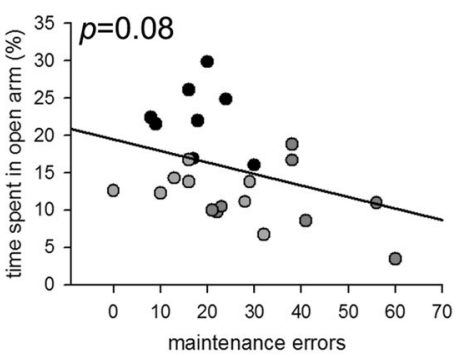

F

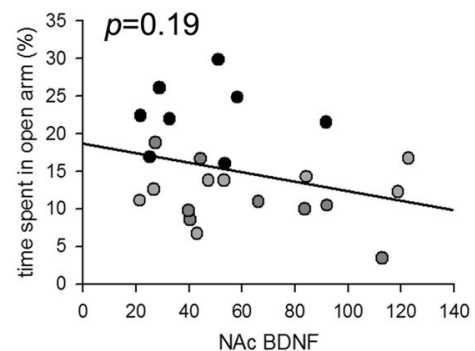

Fig. 4 Anxiety-like symptoms associated with cognitive deficits during spontaneous nicotine withdrawal. Spontaneous nicotine withdrawal produced robust anxiety-like effects, indicated by reduce time spent in open arms, on the first and third day of withdrawal, but were absent 7 days after pump removal (a). In general, a negative association was observed between the time spent in open arms and perseverative errors (b), but not strategy maintenance errors (c). Interestingly, PFC BDNF expression positively correlated with increased time spent in the open arm (d). Conversely, DS BDNF protein expression was negatively associated with this behavioral measure (e). Changes in NAc BDNF expression were not associated with anxiety-like symptoms (f). The strength for all associations was measured using Pearson's $r$ correlations.

memory, and cognitive processes involved in the maintenance of information concerning novel strategies and reward [49, 50] further supports this interpretation. It is possible that nicotine withdrawal-related deficits in multiple component processes of cognitive set-shifting may interfere with the ability to forgo detrimental habits that are formed during nicotine-taking and trigger relapse during early and prolonged abstinence in smokers.

BDNF is a dynamic regulator of activity-dependent plasticity and this neurotrophin is implicated in addiction [31, 51, 52]. Parallel increases in the expression of BDNF exon-IV and exon-IX transcripts in the mPFC of high-dose nicotine withdrawal mice indicated that increased BDNF transcription presumably occurred as a consequence of withdrawal-related alterations in prefrontal activity. These findings align with previous studies that reported increases in PFC BDNF mRNA expression during cocaine withdrawal $[53,54]$. Surprisingly, BDNF protein levels declined in mPFC while an inverse increase was noted in the DS during nicotine withdrawal. Moreover, correlation analysis revealed a negative trend for BDNF protein between the two brain regions. Given the evidence that BDNF is anterogradely transported from cortical neurons to target striatal regions, and that the translation of BDNF by striatal neurons is limited $[55,56]$, higher DS BDNF levels observed in our study could plausibly be linked to increased PFCDS BDNF overflow as a compensatory neuroadaptive response during nicotine withdrawal. Lack of changes in proBDNF protein levels in the PFC and DS during nicotine withdrawal may also suggest that this transport might have occurred only in the mature form of BDNF following the proteolytic cleavage in the PFC. At this point, this interpretation remains speculative and the hypothesis that nicotine withdrawal facilitates prefrontal activity-dependent anterograde transport of BDNF to DS remains to be tested. Although there were subtle differences in the BDNF protein in the PFC and DS of low-dose nicotine withdrawal animals, these did not reach significance. It is possible that perturbations in frontostriatal BDNF expression remained moderate due to lower intensity of withdrawal symptoms, and that BDNF levels returned to baseline levels faster than the high-dose nicotine withdrawal mice. 
$\mathrm{A} \longrightarrow$ sal
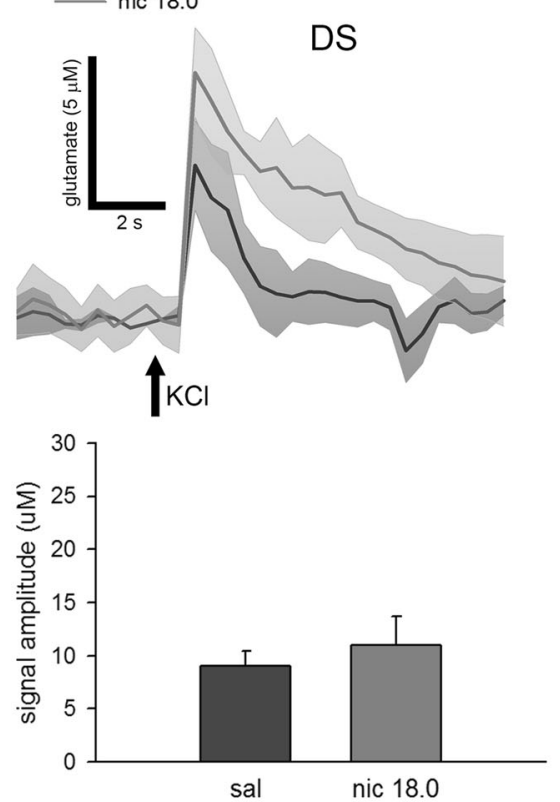

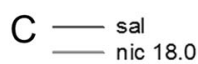
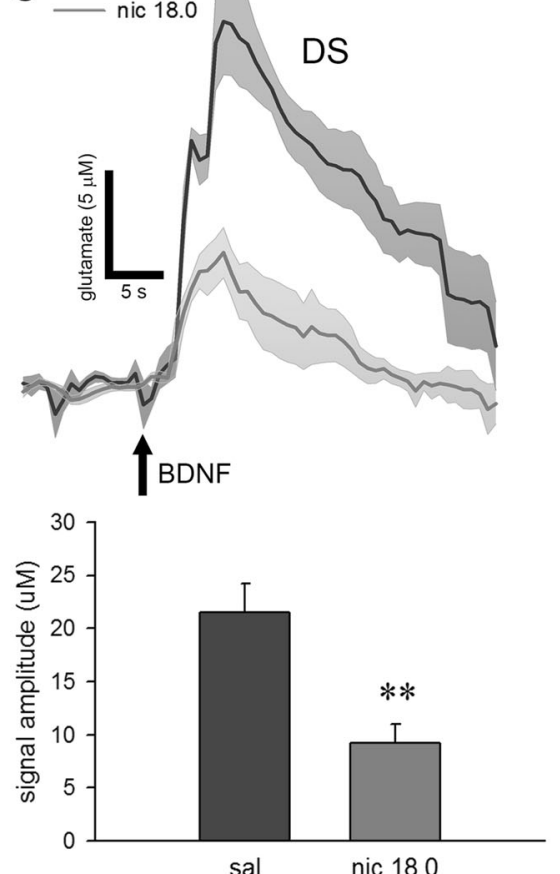

B
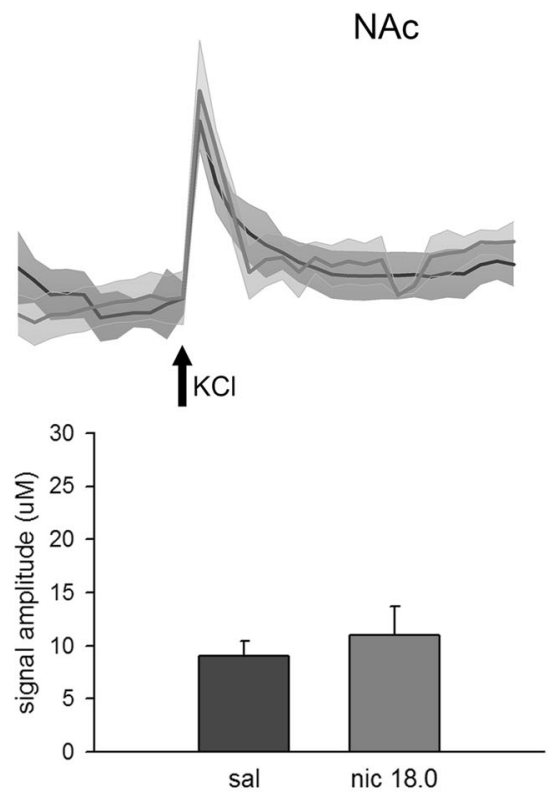

D

NAc

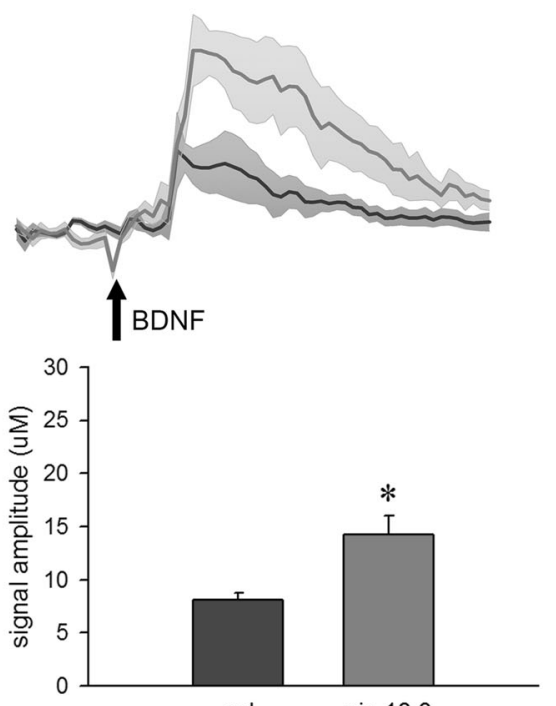

sal

nic 18.0

Fig. 5 In vivo amperometric recordings striatal glutamatergic transmission. Population traces depicting synaptic glutamate release following brief depolarizing pulses of potassium in the DS (a, upper panel) and NAc (b, upper panel) of mice undergoing saline and nicotine (18 mg/kg/ day) withdrawal. Bar charts (lower panel) depict that average glutamate signal amplitudes remained similar between the two groups indicating that nicotine withdrawal neither altered presynaptic release of glutamate in the DS nor NAc. Population traces depicting increases in extracellular glutamate levels following local application of BDNF in the DS (c, upper panel) and NAc (d, upper panel). A significant attenuation of BDNF-evoked glutamate release was observed in the DS of nicotine withdrawal mice compared to the saline-exposed animals treated mice (c, lower panel). On the contrary, glutamate signal amplitudes were significantly higher following local BDNF application in the NAc of nicotine withdrawal mice (d, lower panel). Data are mean \pm SEM ( $n=5 / \mathrm{sal}, n=6 / \mathrm{nic})$ and analyzed using one-way ANOVA. * $p<0.05$; ${ }^{* *} p<0.01$ vs sal.

One of the most interesting findings of our study was that, region-specific variation in BDNF protein levels predicted perseverative and maintenance errors that represent decision processes engaged in early and later stages of cognitive flexibility. MPFC is critically involved in resolving conflicting response tendencies during set-shifting [22]. Moreover, the dorsal and ventral striatal regions are engaged during the different stages of cognitive set reconfiguration [57]. Therefore, region-specific alterations of BDNF protein in the frontostriatal circuits may underlie deficits in specific component processes of cognitive set-shifting. This proposition is supported by previous studies demonstrating that elevations of BDNF in the MPFC induced stimulus-response habits, and that a 
balanced corticostriatal BDNF-trkB signaling maintained flexible goal-directed behaviors [58, 59].

Cognitive control processes including perceptual selection, response biasing, and online maintenance of goal-related information are known to be influenced by affective valence [60]. Aberrant PFC function associated with anxiety is suggested to contribute to deficits in cognitive flexibility [61, 62]. Moreover, reduced BDNF levels in the MPFC were associated with anxiety in early weaned mice [63]. The positive association of withdrawalrelated anxiety with perseverative errors and DS BDNF, and an inverse association of this behavior with PFC BDNF, suggests that early deficits in strategy set-shifting might mostly occur as a consequence of withdrawal-related affective dysfunction and that this relationship is mediated by PFC to striatal BDNF overdrive. A previous study demonstrating that transgenic mice that overexpressed the mature form of BDNF exhibited anxiety-like traits [64] further supports this view. Although NAc is known to be involved in mediating certain aspects of cognitive flexibility and affective information processing [65], absence of any BDNF alterations in this brain region during nicotine withdrawal was surprising. There could be two potential explanations for this. First, it is possible that withdrawal-related alterations in PFC-NAC circuitry are transient and occur during the early stages of withdrawal. Because the tissue samples for biochemical estimations were extracted after the completion of behavioral testing ( 2 weeks after withdrawal), NAc BDNF changes might have returned to baseline levels at that point. Second, we used NAC punches for BDNF protein analysis that included both the shell and core regions and it is possible that the effects of nicotine withdrawal were dissociable between the two regions and became diluted when the whole NAc tissue was analyzed.

Suppression of BDNF-elicited glutamate release observed in the DS of nicotine withdrawal mice could be a consequence of negative feedback inhibition due to prolonged presynaptic trkB activation by endogenous BDNF secreted from glutamatergic afferents. This finding is consistent with our previous work where we reported an inverted-U relationship between BDNF and DS glutamate transmission [32]. Although striatal trkB receptor densities remained unaltered with nicotine withdrawal, synapsin phosphorylation was reduced in the DS synaptosomes isolated from nicotine withdrawal mice. This suggests that diminished glutamate responsivity to BDNF could be linked to inhibition of trkB signaling pathways (see Supplementary Information). Our interpretation is supported by the evidence that BDNF regulates presynaptic glutamate release via MAPK-mediated phosphorylation of synapsin downstream of trkB [66]. Although BDNF and trkB protein levels in the NAc remained similar between saline and nicotine withdrawal mice, opposing effects on glutamate signaling were observed. BDNF-elicited enhanced glutamate release in the NAc of nicotine withdrawal mice. Additionally, unlike DS, the suppression of synapsin phosphorylation was also not observed in the NAc. Taken together, these data suggest that nicotine withdrawal-related frontostriatal circuit adaptations involve dissociable effects of BDNF on DS and NAc glutamate signaling. Striatal glutamate signaling is implicated in cognitive flexibility $[67,68]$ and aberrations in glutamatergic transmission are widely reported during nicotine withdrawal [69-71]. Thus, it is possible that nicotine withdrawal-mediated deficits in cognitive flexiblity may involve BDNF-mediate perturbations in frontostriatal glutamate signling.

There are several limitations of our study that requires some discussion. First, all experiments were conducted in male mice which make it difficult to generalize the behavioral and neurochemical effects of nicotine withdrawal to both sexes. Although we did not observe sex differences in cognitive flexibility performance in our previous study [16], it is possible that nicotine withdrawal might impact executive functions in the male and female mice differentially. Second, the specificity of frontostriatal changes in presynaptic glutamate signaling may be limited. Besides cortex, striatum also receives excitatory glutamatergic inputs from the thalamus, and it is possible that some of the effects of nicotine withdrawal on BDNF-glutamate interactions might have occurred due to perturbations in thalamostriatal circuits. Lastly, all in vivo glutamate recordings were conducted in naïve animals. Although, the withdrawal time points for recording sessions paralleled behavioral experiments when nicotine withdrawal-related deficits were prominent, the glutamate data are insufficient to link glutamate changes to alterations in cognitive flexibility.

In conclusion, our study provides novel insights into the cellular substrates that underlie nicotine withdrawal-related deficits in cognitive flexibility. Specifically, we show that alterations in BDNF signaling in discrete frontostriatal circuits impact distinct components of cognitive set-shifting during nicotine abstinence. Moreover, the interactions between BDNF and presynaptic glutamate signaling dynamics were differentially affected in the DS and NAC with nicotine withdrawal. Further studies are warranted to test the hypothesis that BDNF-mediated perturbations in frontostriatal glutamate networks impose cognitive rigidity in abstinent smokers. Overall, our data point to BDNF playing a larger role in nicotine dependence than previously thought. Whether therapeutic strategies that balance frontostriatal BDNF signaling during abstinence have the potential to restore flexible cognition in smokers and prevent relapse remains to be seen.

\section{FUNDING AND DISCLOSURE}

This work was supported by grants from the National Institute of Health (DA037421 and DA017949). The authors declare no competing interests.

\section{ADDITIONAL INFORMATION}

Supplementary Information accompanies this paper at (https://doi.org/10.1038/ s41386-019-0574-6)

Publisher's note Springer Nature remains neutral with regard to jurisdictional claims in published maps and institutional affiliations.

\section{REFERENCES}

1. De Biasi M, Dani JA. Reward, addiction, withdrawal to nicotine. Annu Rev Neurosci. 2011;34:105-30.

2. Jackson KJ, Muldoon PP, De Biasi M, Damaj MI. New mechanisms and perspectives in nicotine withdrawal. Neuropharmacology. 2015;96:223-34.

3. Koob GF, Le Moal M. Review. Neurobiological mechanisms for opponent motivational processes in addiction. Philos Trans R Soc Lond B Biol Sci. 2008;363:3113-23.

4. Markou A. Review. Neurobiology of nicotine dependence. Philos Trans R Soc Lond B Biol Sci. 2008;363:3159-68.

5. Everitt BJ, Robbins TW. Neural systems of reinforcement for drug addiction: from actions to habits to compulsion. Nat Neurosci. 2005;8:1481-9.

6. Gould TJ. Addiction and cognition. Addict Sci Clin Pract. 2010;5:4-14.

7. Ashare RL, Falcone $M$, Lerman $C$. Cognitive function during nicotine withdrawal: implications for nicotine dependence treatment. Neuropharmacology. 2014;76(Pt B):581-91.

8. Davis JA, James JR, Siegel SJ, Gould TJ. Withdrawal from chronic nicotine administration impairs contextual fear conditioning in C57BL/6 mice. J Neurosci. 2005:25:8708-13.

9. Gould TJ, Wilkinson DS, Yildirim E, Blendy JA, Adoff MD. Dissociation of tolerance and nicotine withdrawal-associated deficits in contextual fear. Brain Res. 2014; 1559:1-10.

10. Ashare RL, McKee SA. Effects of varenicline and bupropion on cognitive processes among nicotine-deprived smokers. Exp Clin Psychopharmacol. 2012;20:63-70.

11. Patterson F, Jepson C, Loughead J, Perkins K, Strasser AA, Siegel S, et al. Working memory deficits predict short-term smoking resumption following brief abstinence. Drug Alcohol Depend. 2010;106:61-64.

12. Semenova S, Stolerman IP, Markou A. Chronic nicotine administration improves attention while nicotine withdrawal induces performance deficits in the 5-choice serial reaction time task in rats. Pharm Biochem Behav. 2007:87:360-8. 
13. Grabski M, Curran HV, Nutt DJ, Husbands SM, Freeman TP, Fluharty $M$, et al. Behavioural tasks sensitive to acute abstinence and predictive of smoking cessation success: a systematic review and meta-analysis. Addiction. 2016; 111:2134-44.

14. Goldstein RZ, Volkow ND. Dysfunction of the prefrontal cortex in addiction: neuroimaging findings and clinical implications. Nat Rev Neurosci. 2011; 12:652-69.

15. Allison C, Shoaib M. Nicotine improves performance in an attentional set shifting task in rats. Neuropharmacology. 2013;64:314-20.

16. Cole RD, Poole RL, Guzman DM, Gould TJ, Parikh V. Contributions of beta2 subunit-containing $\mathrm{nAChRs}$ to chronic nicotine-induced alterations in cognitive flexibility in mice. Psychopharmacology (Berl). 2015;232:1207-17.

17. Ortega LA, Tracy BA, Gould TJ, Parikh V. Effects of chronic low- and high-dose nicotine on cognitive flexibility in C57BL/6J mice. Behav Brain Res. 2013; 238:134-45.

18. Balleine BW, Delgado MR, Hikosaka O. The role of the dorsal striatum in reward and decision-making. J Neurosci. 2007;27:8161-5

19. Vaghi $M M$, Vertes $P E$, Kitzbichler MG, Apergis-Schoute $A M$, van der Flier $F E$, Fineberg NA, et al. Specific frontostriatal circuits for impaired cognitive flexibility and goal-directed planning in obsessive-compulsive disorder: evidence from resting-state functional connectivity. Biol Psychiatry. 2017;81:708-17.

20. Bisonette GB, Powell EM, Roesch MR. Neural structures underlying set-shifting: roles of medial prefrontal cortex and anterior cingulate cortex. Behav Brain Res. 2013;250:91-101.

21. Floresco SB, Ghodes-Sharifi S, Vexelman C, Magyar O. Dissociable roles for the nucleus accumbens core and shell in regulating set-shifting. J Neurosci. 2006; 26:2449-57.

22. Ragozzino ME. The contribution of the medial prefrontal cortex, orbitofrontal cortex, and dorsomedial striatum to behavioral flexibility. Ann NY Acad Sci. 2007; 1121:355-75.

23. Ragozzino ME, Ragozzino KE, Mizumori SJ, Kesner RP. Role of the dorsomedial striatum in behavioral flexibility for response and visual cue discrimination learning. Behav Neurosci. 2002;116:105-15.

24. Brooks JM, Pershing ML, Thomsen MS, Mikkelsen JD, Sarter M, Bruno JP. Transient inactivation of the neonatal ventral hippocampus impairs attentional set-shifting behavior: reversal with an a7 nAChR agonist. Neuropsychopharmacology. 2012;37:2476-86.

25. Placek K, Dippel WC, Jones S, Brady AM. Impairments in set-shifting but not reversal learning in the neonatal ventral hippocampus lesion model of schizophrenia: further evidence for medial prefrontal deficits. Behav Brain Res. 2013;256:405-13.

26. Brigman JL, Rothblat LA. Stimulus specific deficit on visual reversal learning after lesions of medial prefrontal cortex in the mouse. Behav Brain Res. 2008; 187:405-10.

27. Haber SN, Kim KS, Mailly P, Calzavara R. Reward-related cortical inputs define a large striatal region in primates that interface with associative cortical connections, providing a substrate for incentive-based learning. J Neurosci. 2006; 26:8368-76.

28. Stefani MR, Moghaddam B. Activation of type 5 metabotropic glutamate receptors attenuates deficits in cognitive flexibility induced by NMDA receptor blockade. Eur J Pharm. 2010;639:26-32.

29. Jia Y, Gall CM, Lynch G. Presynaptic BDNF promotes postsynaptic long-term potentiation in the dorsal striatum. J Neurosci. 2010;30:14440-5.

30. Jing D, Lee FS, Ninan I. The BDNF Val66Met polymorphism enhances glutamatergic transmission but diminishes activity-dependent synaptic plasticity in the dorsolateral striatum. Neuropharmacology. 2017;112:84-93.

31. Park $H$, Popescu A, Poo MM. Essential role of presynaptic NMDA receptors in activity-dependent BDNF secretion and corticostriatal LTP. Neuron. 2014; 84:1009-22.

32. D'Amore DE, Tracy BA, Parikh V. Exogenous BDNF facilitates strategy set-shifting by modulating glutamate dynamics in the dorsal striatum. Neuropharmacology. 2013;75:312-23

33. Parikh V, Cole RD, Patel PJ, Poole RL, Gould TJ. Cognitive control deficits during mecamylamine-precipitated withdrawal in mice: Possible links to frontostriatal BDNF imbalance. Neurobiol Learn Mem. 2016;128:110-6.

34. Haluk DM, Floresco SB. Ventral striatal dopamine modulation of different forms of behavioral flexibility. Neuropsychopharmacology. 2009;34:2041-52.

35. Monchi O, Petrides M, Petre V, Worsley $\mathrm{K}$, Dagher A. Wisconsin card sorting revisited: distinct neural circuits participating in different stages of the task identified by event-related functional magnetic resonance imaging. J Neurosci. 2001;21:7733-41.

36. Marks MJ, Rowell PP, Cao JZ, Grady SR, McCallum SE, Collins AC. Subsets acetylcholine-stimulated $86 \mathrm{Rb}+$ efflux [125I]-epibatidine binding sites C57BL/6 mouse brain are differentially affected chronic nicotine treatment. Neuropharmacology. 2004;46:1141-57.
37. Benowitz NL, Porchet $H$, Jacob P. Nicotine dependence and tolerance in man: pharmacokinetic and pharmacodynamic investigations. Prog Brain Res. 1989;79:279-87

38. Damaj Ml, Kao W, Martin BR. Characterization of spontaneous and precipitated nicotine withdrawal in the mouse. J Pharm Exp Ther. 2003;307:526-34.

39. Portugal GS, Kenney JW, Gould TJ. $\beta 2$ subunit containing acetylcholine receptors mediate nicotine withdrawal deficits in the acquisition of contextual fear conditioning. Neurobiol Learn Mem. 2008;89:106-13.

40. Aid T, Kazantseva A, Piirsoo M, Palm K, Timmusk T. Mouse and rat BDNF gene structure and expression revisited. J Neurosci Res. 2007;85:525-35.

41. Kenney JW, Florian C, Portugal GS, Abel T, Gould TJ. Involvement of hippocampal jun- $\mathrm{N}$ terminal kinase pathway in the enhancement of learning and memory by nicotine. Neuropsychopharmacology. 2010;35:483-92.

42. Parikh V, Ji J, Decker MW, Sarter M. Prefrontal beta2 subunit-containing and alpha7 nicotinic acetylcholine receptors differentially control glutamatergic and cholinergic signaling. J Neurosci. 2010;30:3518-30.

43. Parikh V, Man K, Decker MW, Sarter M. Glutamatergic contributions to nicotinic acetylcholine receptor agonist-evoked cholinergic transients in the prefrontal cortex. J Neurosci. 2008;28:3769-80.

44. Livingstone PD, Wonnacott S. Nicotinic acetylcholine receptors and the ascending dopamine pathways. Biochem Pharm. 2009;78:744-55.

45. Kalivas PW, Volkow ND. The neural basis of addiction: a pathology of motivation and choice. Am J Psychiatry. 2005;162:1403-13.

46. Kozink RV, Lutz AM, Rose JE, Froeliger B, McClernon FJ. Smoking withdrawal shifts the spatiotemporal dynamics of neurocognition. Addict Biol. 2010;15:480-90.

47. Kolokotroni KZ, Rodgers RJ, Harrison AA. Effects of chronic nicotine, nicotine withdrawal and subsequent nicotine challenges on behavioural inhibition in rats. Psychopharmacology (Berl). 2012;219:453-68.

48. Ashare RL, Hawk LW Jr. Effects of smoking abstinence on impulsive behavior among smokers high and low in ADHD-like symptoms. Psychopharmacology (Berl). 2012;219:537-47.

49. Hill SK, Reilly JL, Ragozzino ME, Rubin LH, Bishop JR, Gur RC, et al. Regressing to prior response preference after set switching implicates striatal dysfunction across psychotic disorders: findings from the B-SNIP study. Schizophr Bull. 2015;41:940-50.

50. Barcelo F, Knight RT. Both random and perseverative errors underlie WCST deficits in prefrontal patients. Neuropsychologia. 2002;40:349-56.

51. Graham DL, Edwards S, Bachtell RK, Dileone RJ, Rios M, Self DW. Dynamic BDNF activity in nucleus accumbens with cocaine use increases self-administration and relapse. Nat Neurosci. 2007;10:1029-37.

52. Russo SJ, Mazei-Robison MS, Ables JL, Nestler EJ. Neurotrophic factors and structural plasticity in addiction. Neuropharmacol. 2009;56(Suppl 1):73-82.

53. Filip M, Faron-Gorecka A, Kusmider M, Golda A, Frankowska M, DziedzickaWasylewska M. Alterations in BDNF and trkB mRNAs following acute or sensitizing cocaine treatments and withdrawal. Brain Res. 2006;1071:218-25.

54. Lu H, Cheng PL, Lim BK, Khoshnevisrad N, Poo MM. Elevated BDNF after cocaine withdrawal facilitates LTP in medial prefrontal cortex by suppressing GABA inhibition. Neuron. 2010;67:821-33.

55. Conner JM, Lauterborn JC, Yan Q, Gall CM, Varon S. Distribution of brain-derived neurotrophic factor (BDNF) protein and mRNA in the normal adult rat CNS: evidence for anterograde axonal transport. J Neurosci. 1997;17:2295-313.

56. Altar CA, Cai N, Bliven T, Juhasz M, Conner JM, Acheson AL, et al. Anterograde transport of brain-derived neurotrophic factor and its role in the brain. Nature. 1997;389:856-60.

57. Sleezer BJ, Hayden BY. Differential contributions of ventral and dorsal striatum to early and late phases of cognitive set reconfiguration. J Cogn Neurosci. 2016;28:1849-64.

58. Gourley SL, Swanson AM, Jacobs AM, Howell JL, Mo M, Dileone RJ, et al. Action control is mediated by prefrontal BDNF and glucocorticoid receptor binding. Proc Natl Acad Sci USA. 2012;109:20714-9.

59. Pitts EG, Li DC, Gourley SL. Bidirectional coordination of actions and habits by TrkB in mice. Sci Rep. 2018;8:4495.

60. Chiew KS, Braver TS. Positive affect versus reward: emotional and motivational influences on cognitive control. Front Psychol. 2011;2:279.

61. Gruber AJ, Calhoon GG, Shusterman I, Schoenbaum G, Roesch MR, O'Donnell P. More is less: a disinhibited prefrontal cortex impairs cognitive flexibility. J Neurosci. 2010;30:17102-10

62. Park J, Moghaddam B. Impact of anxiety on prefrontal cortex encoding of cognitive flexibility. Neuroscience. 2017;345:193-202.

63. Kikusui T, Kanbara N, Ozaki M, Hirayama N, Ida K, Tokita M, et al. Early weaning increases anxiety via brain-derived neurotrophic factor signaling in the mouse prefrontal cortex. Sci Rep. 2019;9:3991.

64. Papaleo F, Silverman JL, Aney J, Tian Q, Barkan CL, Chadman KK, et al. Working memory deficits, increased anxiety-like traits, and seizure susceptibility in BDNF overexpressing mice. Learn Mem. 2011;18:534-44. 
876

65. Floresco SB. The nucleus accumbens: an interface between cognition, emotion and action. Ann Rev Psychol. 2015;66:25-52.

66. Jovanovic JN, Czernik AJ, Fienberg AA, Greengard P, Sihra TS. Synapsins as mediators of BDNF-enhanced neurotransmitter release. Nat Neurosci. 2000;3:323-9.

67. Ding X, Qiao Y, Piao C, Zheng X, Liu Z, Liang J. N-methyl-D-aspartate receptormediated glutamate transmission in nucleus accumbens plays a more important role than that in dorsal striatum in cognitive flexibility. Front Behav Neurosci. 2014;8:304.

68. Palencia CA, Ragozzino ME. The influence of NMDA receptors in the dorsomedial striatum on response reversal learning. Neurobiol Learn Mem. 2004;82:81-89.
69. Cross AJ, Anthenelli R, Li X. Metabotropic glutamate receptors 2 and 3 as targets for treating nicotine addiction. Biol Psychiatr. 2018;83:947-54.

70. Kenny PJ, Gasparini F, Markou A. Group II metabotropic and alpha-amino-3hydroxy-5-methyl-4-isoxazole propionate (AMPA)/kainate glutamate receptors regulate the deficit in brain reward function associated with nicotine withdrawal in rats. J Pharm Exp Ther. 2003;306:1068-76.

71. Liechti ME, Markou A. Interactive effects of the mGlu5 receptor antagonist MPEP and the mGlu2/3 receptor antagonist LY341495 on nicotine self-administration and reward deficits associated with nicotine withdrawal in rats. Eur J Pharm. 2007;554:164-74. 